\title{
How We Make (Ourselves) Believe: \\ Psychological Factors Linked to Belief in (Mis)information About Science
}

\author{
NiCOLE KRAUSE
}

\begin{abstract}
As online communication alters the media ecosystem and changes the way individuals engage with science, concerns about misinformation and a possible rise in science-related misperceptions are increasingly apparent. In this talk, I will review some of the psychological factors that can influence perceptions of (mis)information about science, including the different roles that individual ability and motivation can play in information processing. I will also discuss why it can be difficult to correct false beliefs about science-especially in social media environments - and will review a variety of common and recently-emerging strategies for doing so, some of which are more promising than others.
\end{abstract}

